\title{
Flex Bending Fatigue of Dental Archwires
}

\author{
Janet L. Gbur ${ }^{1}$, Kimaya N. Gupte ${ }^{2}$ and John J. Lewandowski ${ }^{1}$ \\ 1. Case Western Reserve University, Materials Science and Engineering, Cleveland, OH, USA. \\ 2. Case Western Reserve University, Biomedical Engineering, Cleveland, OH, USA.
}

Dental archwires are used to treat patients with malocculusion by producing forces on the teeth to move them into an aligned position. To achieve movement with minimal patient discomfort and provide appropriate formability for clinicians to implement the archwires, the material must be able to accommodate a large range of deflection and endure constant forces without plastically deforming [1]. Shape memory alloy (SMA) development has contributed to the advancement of orthodontic treatment by providing excellent strain recovery and lower stiffness than traditional stainless steel wires while maintaining good formability. Furthermore, nickel-free materials such as beta titanium alloys, with properties between the stainless steels and SMA provides treatment flexibility for the clinicians and an alternative for patients with nickel sensitivity, a concern common in SMA alloys, such as Nitinol. The bending stiffness of these materials in archwires has been well documented in literature; however, little information exists on the fatigue behavior.

Nitinol (NiTi), stainless steel (SS) and beta titanium (Beta Ti) archwires with round and rectangular cross sections were evaluated in tension and fully-reversed (i.e. $\mathrm{R}=-1$ ), flex bending fatigue under ambient conditions at $1 \mathrm{~Hz}$. Cyclic strain was calculated for various mandrel sizes and compared to work by Benini [2]. Scanning electron microscopy was used to identify characteristic features associated with the failure mode and note trends in low cycle fatigue (LCF) and high cycle fatigue (HCF) regimes.

Tension results showed characteristic stress/strain curves for all materials, noting the presence of a stress plateau in the NiTi archwire. Ultimate tensile strength (UTS) and modulus of elasticity were greatest for the SS archwires followed by Beta $\mathrm{Ti}$ then NiTi. Fatigue behavior for all materials displayed improvements in fatigue life with an increase in mandrel size and decrease in cyclic strain. Under LCF/high strain and HCF/low strain regimes, the NiTi exhibited longer cyclic life than the Beta Ti and $\mathrm{SS}$ as seen in Figure 1. Fractography of all materials showed ductile behavior with LCF samples exhibiting rougher features and larger regions of rapid fracture compared to HCF samples as seen in Figure 2.

Tension data was clearly delineated among the materials providing archwire options that are strong and stiff when small amounts of tooth movement are needed (SS), elastic when larger malocclusions exist (NiTi) or an intermediate (Beta Ti). The NiTi wires exhibited the greatest fatigue life across the LCF and HCF regimes. Future work should investigate correlations between the fatigue data and in vivo use [3].

\section{References:}

[1] A Verstrynge, J Van Humbeeck and G Willems, American Journal of Orthodontics and Dentofacial Orthopedics 130 (2006), p. 460.

[2] B Benini, MS Thesis, Case Western Reserve University, (2010), p. 1. 
[3] The authors acknowledge Dr. Martin Palomo, Case School of Dental Medicine, Ryan Siebenaller, Case Western Reserve University and Francesca Fabe, Beaumont School, for their assistance.

Additionally, the authors appreciate the support provided by the Arthur P. Armington Professorship.

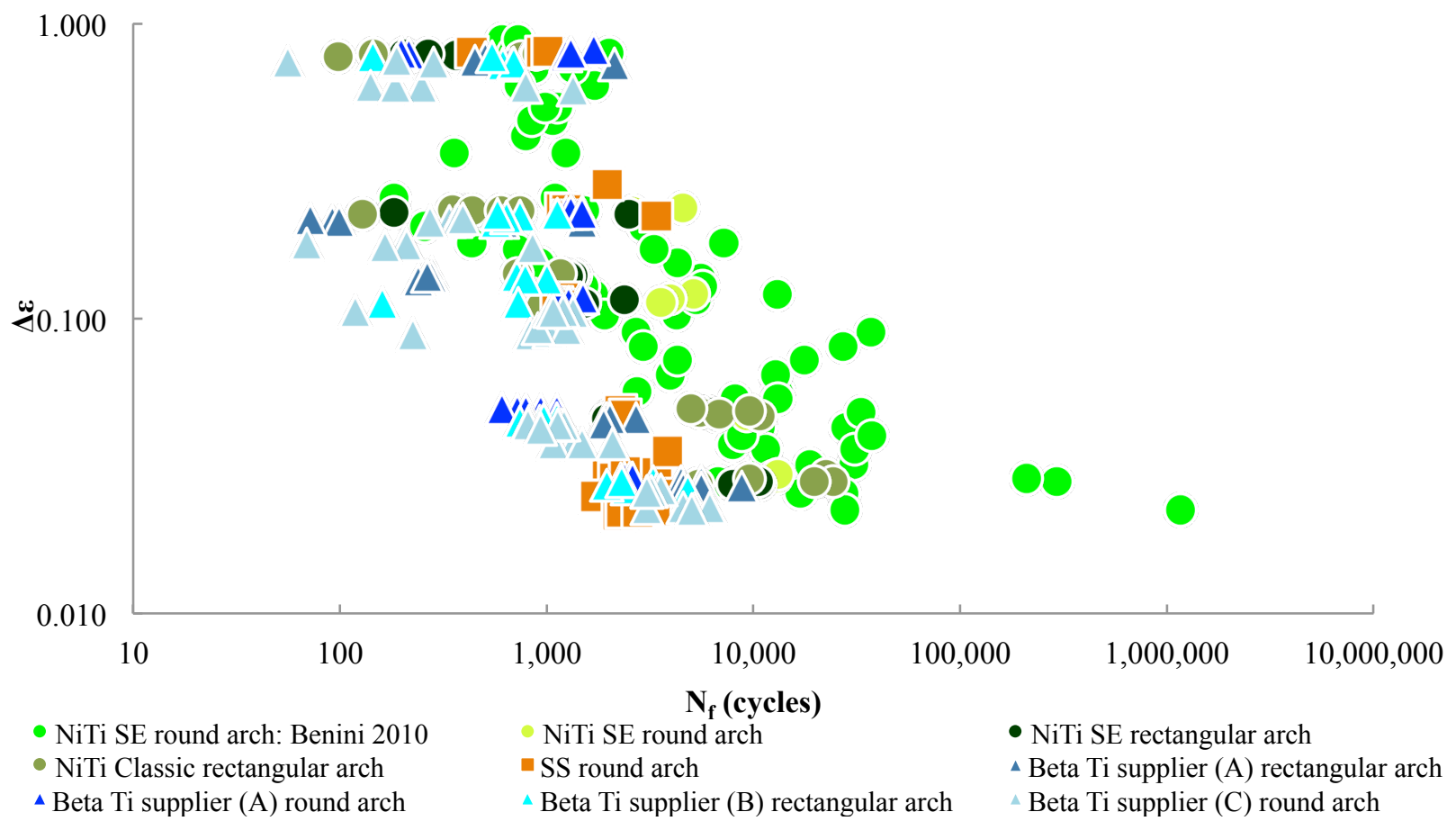

Figure 1. Fatigue data for SS, Beta Ti and NiTi dental archwires.

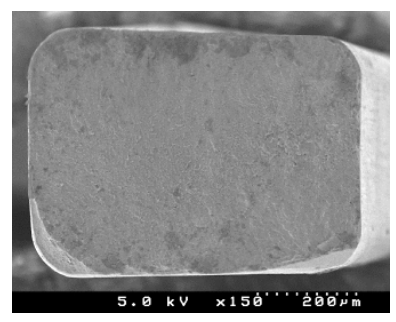

NiTi SE: $\Delta \varepsilon=0.76$ $\mathrm{N}_{\mathrm{f}}=1079$ cycles

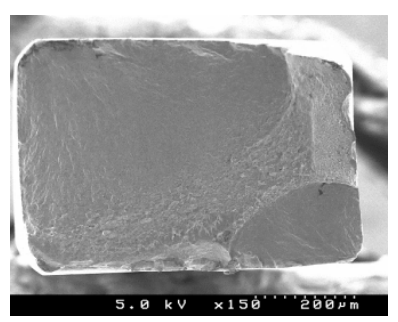

NiTi SE: $\Delta \varepsilon=0.03$ $\mathrm{N}_{\mathrm{f}}=28417$ cycles

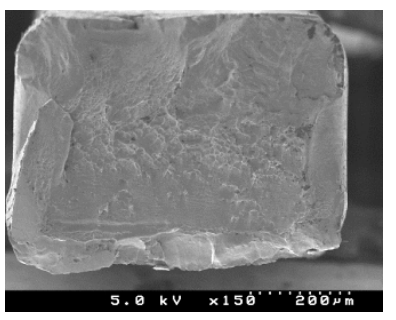

Beta $\operatorname{Ti}(\mathrm{A}): \Delta \varepsilon=0.76$ $\mathrm{N}_{\mathrm{f}}=248$ cycles

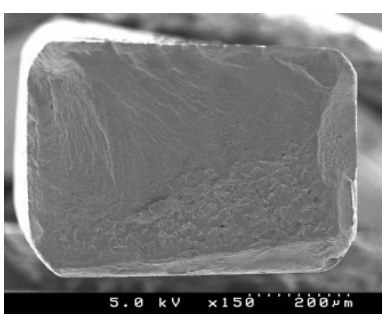

Beta $\operatorname{Ti}(\mathrm{A}): \Delta \varepsilon=0.03$ $\mathrm{N}_{\mathrm{f}}=7765$ cycles

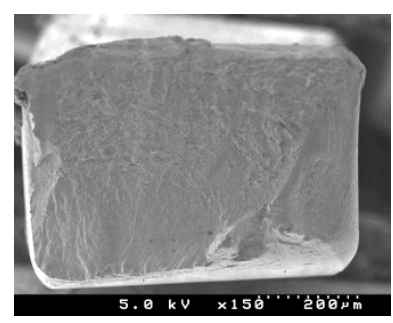

Beta $\operatorname{Ti}(\mathrm{B}): \Delta \varepsilon=0.72$

$\mathrm{N}_{\mathrm{f}}=707$ cycles

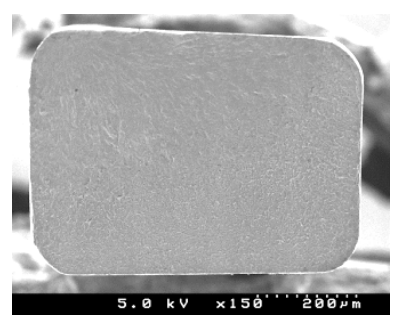

Beta $\operatorname{Ti}(\mathrm{B}): \Delta \varepsilon=0.03$ $\mathrm{N}_{\mathrm{f}}=2954$ cycles

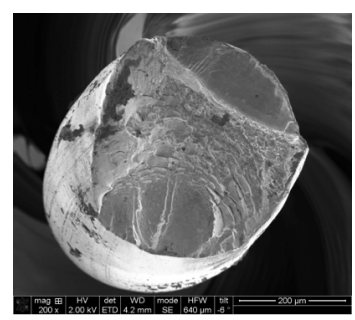

SS: $\Delta \varepsilon=0.80$ $\mathrm{N}_{\mathrm{f}}=943$ cycles

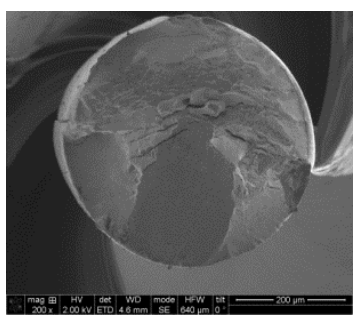

SS: $\Delta \varepsilon=0.03$ $\mathrm{N}_{\mathrm{f}}=3381$ cycles

Figure 2. Fractography of SS, Beta Ti and NiTi dental archwires in low cycle fatigue/high cyclic strain (top row) and high cycle fatigue/low cyclic strain (bottom row). 